\title{
Chlorinated phenols control the expression of the multidrug resistance efflux pump MexAB-OprM in Pseudomonas aeruginosa by interacting with $\mathrm{NalC}$
}

Sudeshna Ghosh, ${ }^{1}$ Claudia M. Cremers, ${ }^{2}$

Ursula Jakob $^{2,3}$ and Nancy G. Love ${ }^{1 *}$

${ }^{1}$ Department of Civil and Environmental Engineering,

University of Michigan, 2350 Hayward Street, 2340 GG

Brown, Ann Arbor, MI 48109-2125, USA.

${ }^{2}$ Department of Molecular, Cellular, and Developmental

Biology, University of Michigan, $830 \mathrm{~N}$-University Ave,

Ann Arbor, MI 48109-1048, USA.

${ }^{3}$ Department of Biological Chemistry, University of

Michigan Medical School, Ann Arbor, MI, USA.

\section{Summary}

NaIC is a TetR type regulator that represses the multidrug efflux pump MexAB-OprM in Pseudomonas aeruginosa. Here we explain the mechanism of NalCmediated regulation of MexAB-OprM. We show that NaIC non-covalently binds chlorinated phenols and chemicals containing chlorophenol side-chains such as triclosan. NalC-chlorinated phenol binding results in its dissociation from promoter DNA and upregulation of NalC's downstream targets, including the MexR antirepressor ArmR. ArmR upregulation and MexR-ArmR complex formation have previously been shown to upregulate MexAB-OprM. In vivo mexB and arm $R$ expression analyses were used to corroborate in vitro NalC-chlorinated phenol binding. We also show that the interaction between chlorinated phenols and NalC is reversible, such that removal of these chemicals restored NalC promoter DNA binding. Thus, the NalC-chlorinated phenol interaction is likely a pertinent physiological mechanism that $P$. aeruginosa uses to control expression of the MexAB-OprM efflux pump.

\section{Introduction}

Pseudomonas aeruginosa is an opportunistic pathogen associated with a wide range of community-acquired and nosocomial infections (Mesaros et al., 2007). P.

Accepted 4 January, 2011. *For correspondence. E-mail nglove@ umich.edu; Tel. (+1) 734764 8495; Fax (+1) 7347644292. aeruginosa infections are responsible for a significant rise in morbidity and mortality in intensive care units (Kerr and Snelling, 2009). Intrinsically resistant to multiple antibiotics, $P$. aeruginosa is a versatile adversary, having the ability to modify and acquire new traits and adapt to diverse environments (Hocquet et al., 2007).

Pseudomonas aeruginosa harbours several chromosomal multidrug resistance (MDR) efflux pumps conferring resistance to a variety of antibiotics (Alekshun and Levy, 2007; Lister et al., 2009). The MexAB-OprM efflux pump has the widest spectrum among these MDR pumps. It mediates the efflux of diverse antibiotics, such as tetracyclines, fluoroquinolones, $\beta$-lactams, chloramphenicol, macrolides, novobiocin, trimethoprim and sulphonamides and biocides such as triclosan (Lister et al., 2009). MexAB-OprM also effluxes quorum sensing molecules (Juhas et al., 2005) and virulence factors (Piddock, 2006). The apparent lack of specificity of MexAB-OprM is intriguing and its natural physiological role unclear (Neyfakh, 1997; Martinez, 2009).

MexAB-OprM has three known transcriptional regulators: MexR, NalD and NalC (Daigle et al., 2007). Among the three regulators, MexR is by far the best studied (Fig. 1A). MexR, which is autoregulated, is transcribed divergently from the same intergenic promoter region as mexAB-oprM (Daigle et al., 2007) (Fig. 1A). MexR's binding to this intergenic region overlaps with promoters for mexR and mexAB-oprM and represses their expression. NaID, another repressor, binds a secondary promoter region of MexAB-OprM and downregulates MexAB-OprM expression as well (Morita et al., 2006). The autoregulator NalC (Fig. 1A) exerts indirect negative control over MexAB-OprM expression by repressing ArmR, an antirepressor of MexR (Daigle et al., 2007). MexR, complexed with ArmR (Fig. 1B), fails to attach to the intergenic promoter region, which results in the overexpression of MexAB-OprM (Wilke et al., 2008). Thus, absence of NalC binding to the promoter region will relieve ArmR repression, thereby promoting ArmR's complex formation with MexR and increasing MexABOprM expression. In addition to the intricate transcriptional control that MexR expression is subjected to, MexR activity is also regulated on the post-translational level. 
A

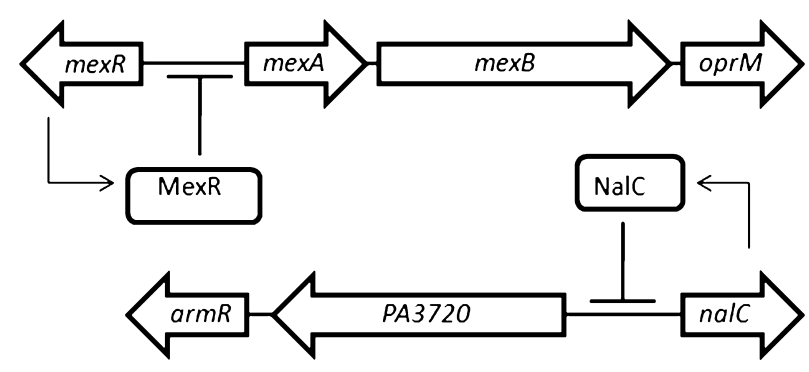

B

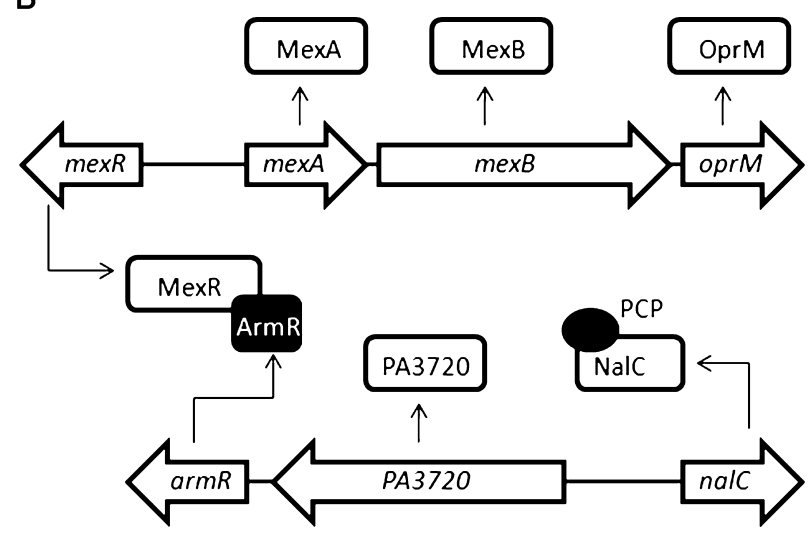

Fig. 1. Regulation of the MexAB-OprM efflux pump in $P$. aeruginosa.

A. PCP not present.

B. PCP present.

$\perp$ denotes binding to the promoter and inactivation of transcription,

$\longrightarrow$ denotes production of protein.

Oxidation of two cysteines in MexR has been shown to cause conformational changes in the protein, preventing it from binding to the promoter DNA region. This redoxmediated control of MexR function leads to the upregulation of the MexAB-OprM operon during oxidative stress conditions both in vitro and in vivo (Chen et al., 2008).

In this paper we show that chlorinated phenols function as NalC effector molecules by reversibly binding to NalC and decreasing its DNA binding affinity, resulting in de-repression of MexAB-OprM expression (Fig. 1B). $\mathrm{NalC}$ is a TetR type regulator with a helix-turn-helix DNA binding domain (DBD) and a ligand binding domain (LBD) (Ramos et al., 2005). The interaction between NalC and chlorinated phenols explains the overexpression of MexAB-OprM and upregulation of mexR, nalC and armR observed by Muller et al. (2007) in P. aeruginosa chemostat cultures in response to treatment with pentachlorophenol (PCP). By using in vitro binding studies, we demonstrate that unlike in response to other organic solvents (Li and Poole, 1999), the PCP-mediated upregulation of the mexAB-oprM operon is not linked to mutations in MexR but is due to the reversible binding of PCPs to
NalC. While chlorinated aromatics including chlorophenols can be produced by natural bacterial and fungal activity (Bengtson et al., 2009), chlorophenols are also commercially produced and are widely present in biocides and disinfectants (Weber et al., 2007). We address the significance of chlorinated phenol-mediated MexABOprM regulation in the light of its environmental occurrences.

\section{Results and discussion}

\section{MexAB-OprM regulators are overexpressed in the presence of $P C P$}

In order to corroborate the results of Muller et al. (2007), who demonstrated PCP-mediated upregulation of mexAB-oprM and its regulators in chemostat cultures, we used quantitative RT-PCR to analyse the expression of $\operatorname{mex} B, \operatorname{mex} R$, armR and nalC in log phase cultures $\left(\mathrm{OD}_{600} \sim 0.3\right)$ with and without $120 \mu \mathrm{M}$ PCP. We observed $\sim 1.5$-fold increases in both mexB and mexR expression in the presence of PCP compared with cultures grown in the absence of PCP (Fig. S1). Expression of armR, a direct target of NalC and MexR's antirepressor, increased greater than 100-fold, while nalC expression increased threefold to fourfold in the presence of PCP. All increases were statistically significant $(P$-values $<0.05)$ based on analysis of variance using MacAnova 5.03. While the fold changes were numerically different, increased expression of these genes in the presence of PCP is consistent with microarray-derived expression data by Muller et al. who reported a threefold to fourfold upregulation of mexABoprM and mexR and 9- and 15-fold increases in armR, $P A 3720$ and nalC in the presence of $\sim 150 \mu \mathrm{M}$ PCP.

\section{PCP does not select for mexR and nalC mutants}

Upregulation of the mexAB-oprM operon in the presence of organic solvents has been previously shown to be connected to mutations in the mexR gene ( $\mathrm{Li}$ and Poole, 1999). To investigate whether a similar mechanism is responsible for our observed increases in the expression of MexR and NalC-regulated genes, we cultured $P$. aeruginosa PAO1 in the presence of PCP and selected colonies on plates containing $150 \mu \mathrm{M}, 1.5 \mathrm{mM}$ and $3.75 \mathrm{mM}$ PCP. None of the colonies analysed from these PCP plates had mutations in either mexR or nalC. This result suggests that upregulation of the mex $R$ and nalC mRNA in the presence of PCP does not require genetic changes in these regulators but functions as a regulated transcriptional response to PCP treatment.

\section{PCP causes dissociation of NalC from its promoter DNA}

Upregulation of both nalC and $a r m R$ coupled with the lack of detectable mutations in nalC led us to hypoth- 


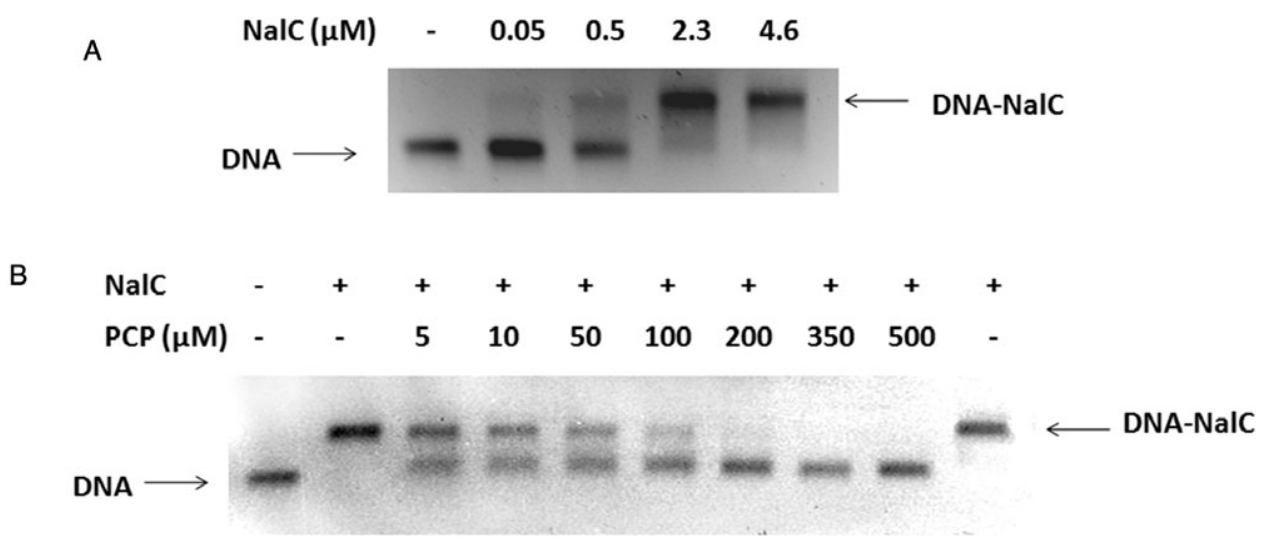

Fig. 2. In vitro analysis of interaction between NalC, promoter DNA and PCP. A. EMSA with $1.2 \mu \mathrm{M}$ promoter DNA and varying concentrations of NalC.

B. EMSA with $1.2 \mu \mathrm{M}$ promoter DNA, $4.6 \mu \mathrm{M}$ NalC and varying concentrations of PCP. Last lane: $70 \mu \mathrm{M}$ NalC and $7.6 \mathrm{mM}$ PCP incubated followed by PCP removal by gel filtration and incubation with DNA. (+): present, (-): absent.

esize that PCP might be directly binding NalC, thus causing the de-repression of its own expression and that of the downstream target armR. ArmR, in turn, would then interact with the repressor MexR, preventing its binding to DNA, and subsequently causing the observed upregulation of mexR and mexAB-oprM. To test our hypothesis we analysed the binding between NalC and promoter DNA in the presence and absence of PCP using electrophoretic mobility shift assay (EMSA) (Fig. 2A). A 262 bp DNA segment encompassing the nalC/PA3720 intergenic region including the start sites of both these genes and the respective promoters, as identified by Cao et al., was used in EMSA (Cao et al., 2004). We found that NalC binding to the promoter DNA region reduced the mobility of the $262 \mathrm{bp}$ DNA segment (Fig. 2A). DNA binding was nearly saturated when $2.3 \mu \mathrm{M} \mathrm{NaIC}$ and $1.2 \mu \mathrm{M}$ promoter DNA were combined.

To assess the effects of PCP on NalC's DNA binding affinity, we tested increasing concentrations of PCP in the presence of $4.6 \mu \mathrm{M} \mathrm{NalC}$ and $1.2 \mu \mathrm{M}$ promoter DNA. As shown in Fig. 2B, the presence of low micromolar concentrations of $\mathrm{PCP}$ was sufficient to significantly decrease the binding affinity of NalC to DNA. At concentrations of PCP above $200 \mu \mathrm{M}$, no binding of NalC to DNA was observed (Fig. 2B). These results are consistent with the conclusion that PCP acts as a ligand of $\mathrm{NalC}$ and prevents $\mathrm{NalC}$ from binding DNA.

To exclude the possibility that PCP caused any irreversible modifications of $\mathrm{NalC}$, thus inactivating the DNA binding protein, we incubated $70 \mu \mathrm{M}$ NalC with $7.6 \mathrm{mM}$ PCP for $1 \mathrm{~h}$. This PCP concentration was sufficient to prevent NalC binding to DNA. Following this incubation, the PCP-NalC mixture was filtered through an Illustra NAP-5 column. The Sephadex gel filtration matrix allows for re-equilibration of protein and ligand and has been used to study reversible protein-ligand binding in the Hummel and Dreyer method (Cann and Hinman, 1976). Following gel filtration, binding between NalC and DNA was completely restored (Fig. 2B, last lane), demonstrating that exposure of NalC to PCP did not permanently change NalC's DNA binding affinity. We concluded from these results that PCP reversibly interacts with NalC to prevent it from binding DNA.

\section{Dichlorophenol, trichlorophenol and triclosan bind NalC}

To assess the ligand specificity of $\mathrm{NalC}$, we examined chemicals structurally similar to PCP for their ability to bind NalC and prevent DNA binding. We tested phenol, 2,4-dichlorophenol (DCP) and 2,4,6-trichlorophenol (TCP) and the phenol-based disinfectant triclosan, which has a monochlorophenol group (Table 1). As before, we assessed binding of $4.6 \mu \mathrm{M}$ NalC to $1.2 \mu \mathrm{M}$ promoter DNA in the presence of these chemicals. While phenol, even at $20 \mathrm{mM}$, did not affect NalC-DNA binding (Fig. 3A), we found that both DCP and TCP diminished the apparent binding affinity of NalC to DNA, similar to the results observed with PCP. In contrast to PCP, however, significantly higher concentrations of DCP and TCP were required to prevent NalC binding to DNA. At $1 \mathrm{mM}$, DCP had minimal effects on NalC's DNA binding while TCP at this concentration partially interfered with NalC-DNA binding. Triclosan also required higher concentrations than PCP to abolish NalC-DNA binding. At $215 \mu \mathrm{M}$, triclosan did not affect NalC-DNA binding, while presence of $430 \mu \mathrm{M}$ triclosan nearly completely prevented NalC's binding to DNA (Fig. 3B). As shown for PCP, DNA binding of NalC was largely restored upon removal of the ligands by dialysis (data not shown). Our 
Table 1. Chemicals tested for binding to NalC using EMSA.

Binding to 4.6 $\mu \mathrm{M}$ NalC
No binding with $20 \mathrm{mM}$
Yes (complete at $9 \mathrm{mM}$ )
Yes (partial at 2.5 mM)

$\mathrm{pK}_{\mathrm{a}}$ and $\log \mathrm{K}_{\mathrm{ow}}$ values are from ChemIDplus (United States National Library of Medicine).

$\mathrm{K}_{\mathrm{ow}}$, octanol-water partition coefficient. It is the ratio of the concentration of the chemical in octanol and in water. Octanol is used as a surrogate for natural organic matter.

findings that the chlorinated phenol derivatives prevent NalC's binding to the promoter region in vitro were in excellent agreement with in vivo expression studies of armR and mexB (Fig. 3C). We found that armR and $m e x B$ transcription levels were reproducibly increased in the presence of DCP, TCP and triclosan, but not in the presence of phenol. These results strongly suggest that chlorination of the phenol is an important characteristic of NalC ligands.

Chlorophenols are weak acids. While PCP shows a $\mathrm{pK}_{\mathrm{a}}$ of 4.74 indicating that it is almost completely de-protonated under our assay conditions $(\mathrm{pH} 7.8), \mathrm{DCP}$ shows a $\mathrm{pK}_{\mathrm{a}}$ of 7.8 and is predicted to be about $50 \%$ de-protonated. We were curious about the relative strength of the protonated versus the de-protonated forms in interacting with NaIC and abolishing DNA binding, which might explain the observed differences in relative affinity of the ligands. We found that binding of NalC to DNA was relatively unaffected in the $\mathrm{pH}$ range of 6-9 (Fig. 3D). Similarly, the effects of PCP on NalC's binding affinity were $\mathrm{pH}$-independent in the chosen $\mathrm{pH}$ range where PCP is largely de-protonated. In stark contrast, however, DCP at pH below 8 (primarily protonated) completely prevented NalC-DNA binding (Fig. 3D). NalCDNA binding increased in the presence of DCP with increasing $\mathrm{pH}$ values, indicating that the protonated form has higher affinity for NalC. These results suggest that the protonation state of the phenol alone cannot explain NalC binding affinities.

\section{Thermodynamic stability of NalC}

The regulatory mechanism of most TetR family members involves the binding of ligands to their LBD, which in turn induces the dissociation of DNA from their DBD. A widely accepted explanation for TetR's allosteric mechanism is that the protein assumes two distinct structures in the ligand-free and ligand-bound states, one that binds DNA and the other that does not (Orth et al., 2000; Ramos et al., 2005). Recently, a different mechanism was proposed by Reichheld et al. (2009), who suggested that the ligand-free TetR has a flexible DBD, arising from a lack of interaction between the DBD and the LBD, and that this flexibility of the DBD is important for DNA binding. The authors then showed that the ligand-free TetR unfolds in a three-state manner, with the unfolding of the DBD preceding the unfolding of the LBD. Binding to the ligand tetracycline increased cooperativity between the two domains of TetR, resulting in rigidification of the DBD and a single cooperative unfolding transition. Based on these data, Reichheld et al. suggested that lack of flexibility of the DBD in the ligand-bound state is responsible for its failure to bind DNA. 

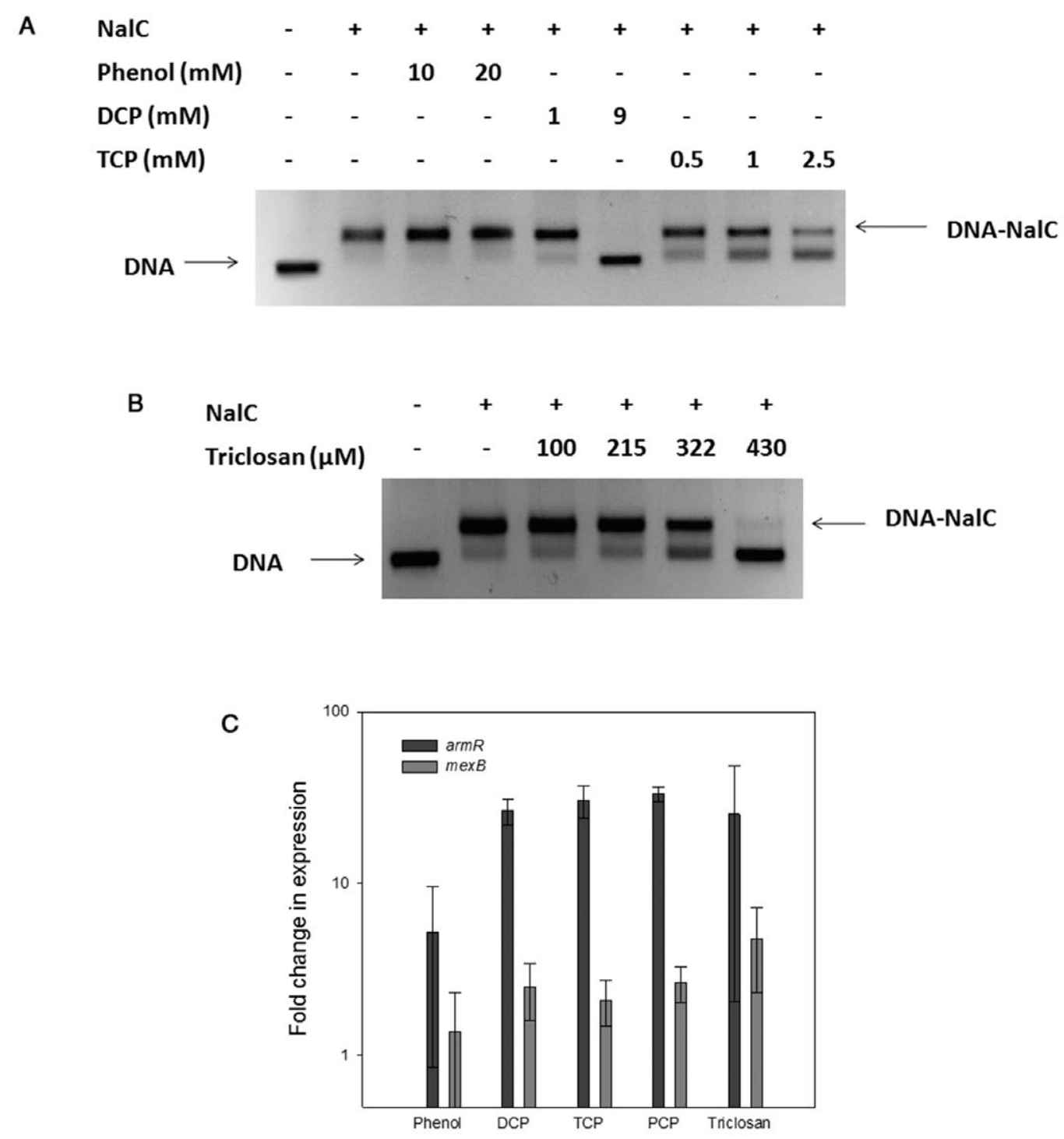

D

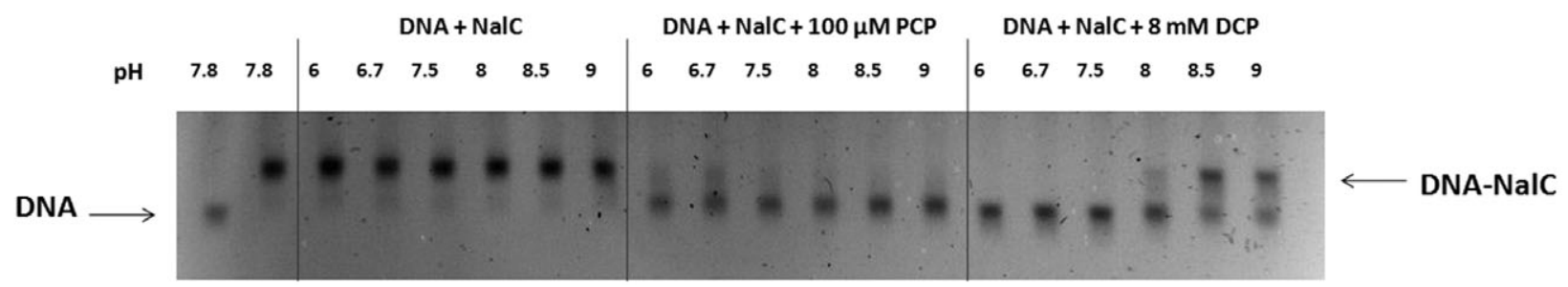

Fig. 3. Interaction of phenolics with NalC.

A. EMSA with $1.2 \mu \mathrm{M}$ promoter DNA, $4.6 \mu \mathrm{M}$ NalC and varying concentrations of phenol, DCP and TCP.

B. EMSA with $1.2 \mu \mathrm{M}$ promoter DNA, $4.6 \mu \mathrm{M} \mathrm{NalC}$ and varying concentrations of triclosan.

C. Fold change in expression of $a r m R$ and mexB normalized to $r p s L$ after addition of $5 \mathrm{mM}$ phenol, $300 \mu \mathrm{M}$ DCP, $400 \mu \mathrm{M} \mathrm{TCP}, 120 \mu \mathrm{M}$ PCP or $100 \mu \mathrm{M}$ triclosan to log phase batch cultures. Bars represent means from three separate batch cultures and error bars represent standard deviations about the mean.

D. EMSA with $1.2 \mu \mathrm{M}$ promoter DNA and $4.6 \mu \mathrm{M}$ NalC. PCP and DCP were used as indicated. $\mathrm{pH}$ was varied between 6 and 9.

$(+)$ : present, (-): absent. 
To monitor the conformational rearrangements of NalC upon ligand binding and its stability in the absence and presence of $\mathrm{PCP}$, we conducted Far-UV circular dichroism (CD) measurements of $\mathrm{NalC}$ as read-out for changes in its secondary structure. We found that in the presence of PCP, NalC gained helicity, suggesting significant structural rearrangements in the LBD upon PCP binding (Fig. 4A). Thermal transition data fitted with sigmoidal curves in SigmaPlot 10 show that the apparent median melting temperature of $\mathrm{NalC}$ also increased from $59^{\circ} \mathrm{C}$ in the absence of $\mathrm{PCP}$ to $78^{\circ} \mathrm{C}$ in the presence of $\mathrm{PCP}$, confirming that PCP binds to NalC, hence the increased stability (Fig. 4B). Upon removal of PCP, NalC behaved like the ligand-free protein, providing further support for the reversibility of NalC-PCP binding. Incubation of NalC in presence of $1.25 \mathrm{mM}$ phenol, a ligand that does not bind NalC, did not affect the structure of NalC (Fig. S2). To further exclude that non-specific effects of PCP might be responsible for the observed changes in NalC's structure and thermal stability, we analysed the CD spectrum and thermal transition of the redox-sensitive chaperone Hsp33 in the presence of $400 \mu \mathrm{M} \mathrm{PCP}$ (Fig. S3). Hsp33 is a two-domain protein containing a meta-stable linker region, and is not known to have ligand binding properties (Graf et al., 2004). As shown in Fig. S3, we were unable to detect any effect of PCP on the structure or thermal stability of Hsp33. These results strongly suggest that the binding of PCP to NalC is specific and increases the stability of the regulator. In contrast to the reported results on TetR, however, ligand-free NalC showed a classical two-state transition whereas the unfolding of PCP-bound NalC was non-cooperative (Fig. 4B), suggesting that the effects of ligand binding might differ among the members of this large protein family. However, neither one of the transitions was fully reversible, precluding us from precisely assessing the role of ligand binding on the stability of NalC.

\section{Significance of NalC activation by chlorinated phenols}

Our observation that NalC expression is induced by chlorinated phenols leads us to speculate on its natural physiological significance. Halogenated organics are naturally present in soil (Myneni, 2002). Different chlorinated phenols, particularly chlorinated methoxyphenols, have been detected in pristine river waters in the ppb $\left(\mu \mathrm{g} \mathrm{I}^{-1}\right)$ concentration range (Michalowicz et al., 2008). We looked at armR expression in the presence of varying concentrations of PCP, and found that concentrations as low as $40 \mathrm{ppb}$ of PCP significantly increased levels of expression of armR in $P$. aeruginosa (data not shown). Many soil microorganisms, including bacteria and fungi, harbour halogenases and haloperoxidases and potentially generate organohalogens (Bengtson et al., 2009; Wagner et al.,
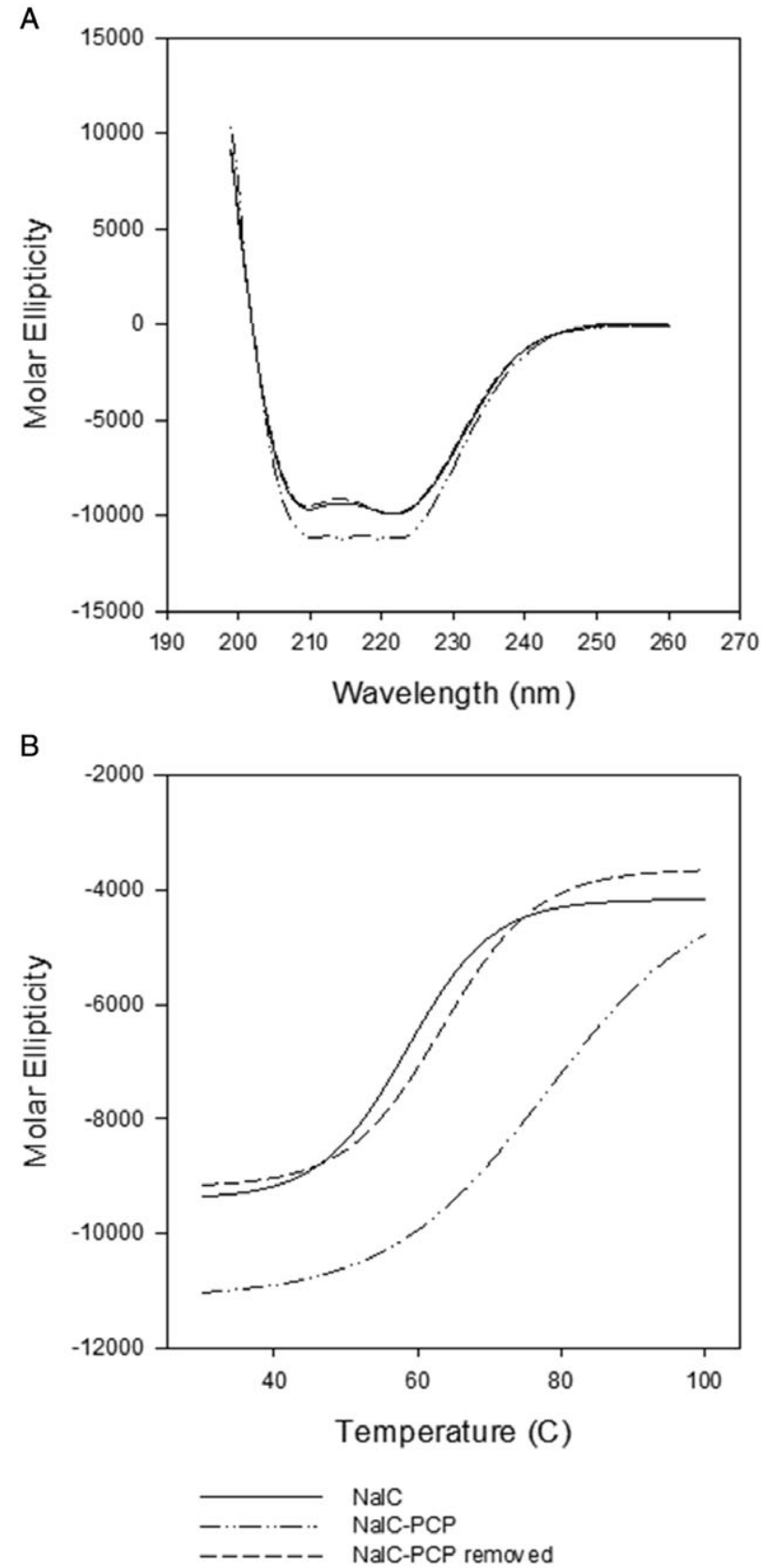

Fig. 4. Effect of PCP on the thermodynamic stability of NalC. A. CD spectra of NalC (straight line), NalC bound to PCP (dashed and dotted line) and NalC incubated with PCP followed by PCP removal by gel filtration (dashed line).

B. Thermal transitions curves for NaIC (straight line), NalC bound to PCP (dashed and dotted line) and NalC incubated with PCP followed by PCP removal by gel filtration (dashed line).

The samples were heated with a rate of $1^{\circ} \mathrm{C} \mathrm{min}-1$ and the CD signal at $222 \mathrm{~nm}$ was monitored. The thermal transitions are not completely reversible.

2009). These observations suggest that $P$. aeruginosa, which is also found in soil (Schobert and Tielen, 2010), is likely to encounter chlorinated organics including chlorophenols in its natural habitat and may have evolved 
NalC-mediated efflux to protect itself. While addition of $150 \mu \mathrm{M}$ PCP to logarithmic phase batch cultures of $P$. aeruginosa PAO1 did not affect its growth rate (Fig. S4A), strains lacking the functional mexB or arm $R$ gene showed reduced growth in the presence of PCP (Fig. S4B and C). Curiously enough, $P$. aeruginosa also harbours a functional chloroperoxidase (Song et al., 2006).

Apart from naturally produced chlorinated phenols, $P$. aeruginosa is likely to be exposed to these chemicals also because of human activities. Chlorophenols, such as 2-chlorophenol, 2,4-DCP, 2,4,6-TCP and PCP have long been used as biocides and wood preservatives and can contaminate soil (McLellan et al., 2007). Chlorination of drinking water also results in the production of minor quantities of chlorophenols (Ge et al., 2008). Both are environments that harbour $P$. aeruginosa (Mena and Gerba, 2009; Schobert and Tielen, 2010). Another environment where $P$. aeruginosa may be exposed to chlorophenols is health-care units (Weber et al., 2007). Triclosan is an antimicrobial used both in health-care units and in a wide variety of household products (Fiss et al., 2007). Other examples of chlorophenol-based disinfectants include chloroxylenol and ortho-benzyl-parachlorophenol (Rutala et al., 2008). The effect of low doses of different halogenated phenols in these environments on $P$. aeruginosa survival, proliferation and other characteristics such as resistance and virulence remains a pertinent question.

\section{Conclusion}

We show here that chlorinated phenols interact with the transcriptional regulator NaIC of the MexAB-OprM MDR efflux pump to control its expression. NalC binding to chlorinated phenols results in de-repression of NaIC and ArmR. Increased expression of ArmR, a MexR antirepressor, results in MexR-ArmR complex formation (Wilke et al., 2008) and alleviates MexR-mediated repression of MexAB-OprM. We demonstrated that NalC-chlorinated phenol binding is fully reversible as NalC regained its DNA binding activity once chlorophenols were removed. We found that triclosan also reversibly binds to NalC. This observation expands the range of chemicals that potentially induce NalC to include chemicals with chlorophenol side-chains. It will now be interesting to determine the precise ligand recognition mechanism that is used by NalC.

As we continue to use and release various purportedly toxic or benign chemicals, it is important for us to understand their full potential in impacting the biosphere. Now we know that antibiotics do not only have antagonistic roles (Linares et al., 2006) and that mechanisms of resistance to antibiotics did not necessarily evolve in response to antibiotics (Piddock, 2006). Similarly, transcriptional regulators are not simple on/off switches, and responses are modulated by the intensity of signalling molecules as well as the different regulatory pathways that intersect to produce a functional organism (Cases and de Lorenzo, 2005). Our observation that chlorinated phenols induce a MDR efflux pump regulator fits these paradigms.

\section{Experimental procedures}

\section{Bacterial strains and growth conditions}

Wild-type $P$. aeruginosa strain PAO1 was obtained from H. Schweizer (Schweizer, 1998). PAO1 was grown in Luria-Bertani (LB) medium at $37^{\circ} \mathrm{C}$ unless otherwise stated. Escherichia coli BL21(DE3) [B834 derivative, $\mathrm{F}{ }^{-}$ompThsd $S_{B}\left(r_{B}^{-} m_{B}^{-}\right)$gal dcm (DE3), Novagen, EMD Biosciences, San Diego, CA, USA] with the pet-15b plasmid (expression vector with $\mathrm{N}$-terminal $\mathrm{His} \cdot \mathrm{Tag}^{\circledR} \mathrm{Ap}^{\mathrm{r}}$, Novagen) was grown on LB with $50 \mu \mathrm{g} \mathrm{ml}^{-1}$ of ampicillin at $37^{\circ} \mathrm{C}$.

\section{Chemicals}

Pentachlorophenol, 2,4-dichlorophenol, 2,4,6-trichlorophenol, and triclosan were purchased from Sigma-Aldrich (Table 1). PCP stock was made at $10 \mathrm{mg} \mathrm{ml}^{-1}$ in $36 \mathrm{mM}$ $\mathrm{NaOH}$. DCP stock was made at a concentration of $5 \mathrm{mg} \mathrm{m}^{-1}$ in Tris buffer ( $\mathrm{pH} \mathrm{7.8).} \mathrm{TCP} \mathrm{stock} \mathrm{was} \mathrm{made} \mathrm{at} 5 \mathrm{mg} \mathrm{ml}^{-1}$ in $36 \mathrm{mM} \mathrm{NaOH}$. Triclosan stock was made at $1.25 \mathrm{mg} \mathrm{ml}^{-1}$ in $50 \%$ solution of $36 \mathrm{mM} \mathrm{NaOH}$. Concentrations of DCP and TCP used in experiments were verified using HPLC.

\section{Gene expression analysis using qRT-PCR}

Pseudomonas aeruginosa PAO1 was grown in minimal medium (Muller et al., 2007) with and without $120 \mu \mathrm{M}$ PCP. Triplicate batch cultures were used for both growth conditions. Samples were collected in duplicate during logarithmic growth phase $\left(\mathrm{OD}_{600} \sim 0.3\right)$, immediately treated with RNAprotect Bacterial Reagent (Qiagen, Valencia, CA, USA) and stored at $-80^{\circ} \mathrm{C}$ for RNA extraction. Gene expression was also analysed in the presence of $300 \mu \mathrm{M}$ DCP, $400 \mu \mathrm{M}$ TCP and $100 \mu \mathrm{M}$ triclosan. PCP $(120 \mu \mathrm{M})$ and phenol $(5 \mathrm{mM})$ were used as positive and negative control respectively. Each condition was tested in triplicate. For this experiment $P$. aeruginosa PAO1 was grown in LB to early logarithmic growth phase $\left(\mathrm{OD}_{600} \sim 0.2\right)$, when chemicals were added. Cells were harvested at $\mathrm{OD}_{600} \sim 0.6$ (still in logarithmic growth phase), immediately treated with RNAprotect Bacteria Reagent and stored at $-80^{\circ} \mathrm{C}$. RNA was extracted using the RNeasy Mini Kit (Qiagen). DNase I, Amplification Grade (Invitrogen Life Science, Carlsbad, CA, USA) was used for DNase digestion prior to cDNA synthesis using the SuperScript II RT kit (Invitrogen Life Science). Random hexamer primers were used for cDNA synthesis. Multiple aliquots of CDNA were stored at $-20^{\circ} \mathrm{C}$ for RT-PCR analysis.

Primers used in qRT-PCR for mexR, mexB, armR and nalC genes are listed in Table 2. Housekeeping genes, nadB and $r p s L$ were used as controls. Quantitative PCR was performed 
Table 2. Primers for RT-qPCR.

\begin{tabular}{lll} 
Name & Sequence $\left(5^{\prime} \rightarrow 3^{\prime}\right)$ & Reference \\
\hline nalC & F: CCT CAC ATG GAC GAG GAA AC & This study \\
armR & R: AGG TAG CAG GCG ATG ATG TC & Cao et al. (2004) \\
mexR & F: CCT GAA CAC TCC GCG CAA C & \\
mexB & F: GAG CTC GCC GTA GAG GTC C & This study \\
nadB & R: AGG CAC TGG TCG AGG AGA T & Muller et al. (2007) \\
rpsL & F: GTG TTC GGC TCG CAG TAC TC & Muller et al. (2007) \\
& R: AC CGT CGG GAT TGA CCT TG & This study \\
\hline
\end{tabular}

in a Mastercycler ep realplex thermocycler (Eppendorf, Hauppauge, NY, USA) using the Power SYBR Green Master Mix (Applied Biosystems, Foster City, CA, USA). PCR conditions and primer concentrations were optimized to eliminate the formation of primer-dimers and non-specific products. All qPCR analyses were performed in triplicate.

\section{Selection of colonies with high PCP tolerance}

To test if PCP selected for $P$. aeruginosa PAO1 with mutations in regulatory genes, colonies grown in medium containing PCP were screened for mutations. Briefly, cultures were grown overnight with $120 \mu \mathrm{M}$ PCP in LB broth, transferred into fresh PCP-containing LB broth and grown to mid-log phase. Logarithmic phase cultures were 10-fold serially diluted in $10 \mathrm{mM}$ phosphate buffer saline $(\mathrm{pH} \sim 7.5)$ and $100 \mu$ c cultures were plated onto LB agar containing PCP at concentrations of $150 \mu \mathrm{M}, 1.5 \mathrm{mM}, 3.75 \mathrm{mM}$ and $6 \mathrm{mM}$. No growth was observed at $6 \mathrm{mM}$ PCP. Colonies were randomly picked from plates containing $150 \mu \mathrm{M}, 1.5 \mathrm{mM}$ and $3.75 \mathrm{mM}$ PCP (10 colonies per PCP concentration) and analysed for mutations in mexR and nalC. Colony PCR was used for amplification of the entire length of these genes. Primers used for amplifying mexR were forward 5'-CATTAGGTT TACTCGGCCAAACC-3' and reverse 5'-CGCCAGTAAGC GGATACCTG-3' (Daigle et al., 2007) and nalC were forward 5'-GAATGAAGCGGAAGTGCTTGC-3' and reverse 5'-CGA GATCCACCTCACCGAAC-3'(Cao et al., 2004). Amplicons were sequenced at the DNA Sequencing Core facility at the University of Michigan (Ann Arbor).

\section{Expression and purification of $\mathrm{NalC}$}

Wild-type nalC was PCR amplified from $P$. aeruginosa PAO1 using forward primer $5^{\prime}$-GTGTGTAAGGCATATGAACGATGC TTCTCC-3' (Ndel site underlined) and reverse primer $5^{\prime}-T$ TCGTATTGGATCCACCTCACCGAACTGC-3'(BamHI site underlined), cloned into pET-15b vector containing the $6 \times$ His tag (EMD Biosciences) and transformed into $E$. coli BL21(DE3) (EMD Biosciences). BL21(DE3) with nalC was grown to $\mathrm{OD}_{600}$ of $\sim 0.6$ and induced with $1 \mathrm{mM}$ IPTG for $3 \mathrm{~h}$. Cells were pelleted, resuspended in phosphate buffer (40 mM KH $\mathrm{PO}_{4}, 200 \mathrm{mM} \mathrm{KCl}, \mathrm{pH}$ 7.5) and treated with Halt Protease Inhibitor Cocktail (Thermo Fisher Scientific, Rock- ford, IL, USA). Cells were lysed in the French press. $6 \times$ HisNalC was purified using a bench-top process using the HisPur Cobalt Resin (Thermo Fisher Scientific) following the manufacturer's protocol. NalC was eluted with a $100 \mathrm{mM}$ imidazole buffer (50 mM KH $\mathrm{KO}_{4}, 300 \mathrm{mM} \mathrm{NaCl}, \mathrm{pH}$ 7.4). Purity of $6 \times$ His-NalC was assessed using SDS-PAGE analysis on a $14 \%$ Tris-glycine gel (Invitrogen). $6 \times$ His tag removal was performed using Restriction Grade Thrombin (EMD Chemicals) followed by elution through the HisPur Cobalt Resin. The cleaved protein was concentrated and loaded onto a HiLoad 26/60 Superdex 75 Prep grade column (GE Healthcare, Piscataway, NJ, USA) equilibrated with HEPES buffer (40 mM HEPES, $100 \mathrm{mM} \mathrm{KCl,} \mathrm{pH} \mathrm{7.8).} \mathrm{Peak} \mathrm{fractions}$ were analysed for the presence of pure NalC using SDSPAGE. NalC-containing fractions were pooled and concentrated to $2 \mathrm{mg} \mathrm{ml}^{-1}$. Protein purity was $>97 \%$ as assessed by SDS-PAGE analysis.

\section{Electrophoretic mobility shift assay}

The nalC/PA3720 intergenic promoter region (Cao et al., 2004) was amplified using primers 5'-AGGCATCGATAT CCAACAGG-3' and 5'-GGGAGAAGCATCGTTCAT-3' and amplification products were purified using QIAquick PCR Purification Kit (Qiagen, Valencia, CA, USA). EMSA was set up with purified NaIC, promoter DNA and varying concentrations of chemicals in a $10 \mathrm{mM}$ Tris-HCl binding buffer $(\mathrm{pH} 7.8)$ containing $1 \mathrm{mM}$ EDTA, $100 \mathrm{mM} \mathrm{KCl}, 5 \% \mathrm{v} / \mathrm{v}$ glycerol, $0.1 \mathrm{mM}$ DTT, $0.01 \mathrm{mg} \mathrm{ml}^{-1}$ BSA (Hellman and Fried, 2007). The NalC protein was pre-incubated with the respective chemicals in the $10 \mathrm{mM}$ Tris-HCl buffer for $1 \mathrm{~h}$. Then, DNA was added and incubation was continued for another $45 \mathrm{~min}$. Samples were run on $0.8 \%$ agarose gels in $1 \times$ TAE at $100 \mathrm{~V}$ at room temperature and stained with SYBR Safe DNA Gel Stain (Invitrogen, Carlsbad, CA, USA). DNA bands were visualized using a Dark Reader Transilluminator (Clare Chemical Research).

\section{NAP-5 filtration and dialysis}

PCP-NalC mixtures were filtered through an Illustra NAP-5 column (GE Healthcare Lifesciences, Piscataway, NJ, USA) packed with Sephadex ${ }^{\mathrm{TM}}$ G-25 and equilibrated with 
PCP-free $10 \mathrm{mM}$ Tris- $\mathrm{HCl}$ binding buffer to remove any noncovalently bound PCP. Similarly, DCP, TCP or triclosan were removed from $\mathrm{NaIC}$ by dialysis using a regenerated cellulose Spectra/Por membrane with an $8 \mathrm{kDa}$ cut-off (Spectrum Laboratories). TCP and triclosan were dialysed overnight, while DCP was dialysed for 2 days.

\section{Circular dichroism spectroscopy}

NalC was diluted to $8.7 \mu \mathrm{M}\left(0.2 \mathrm{mg} \mathrm{ml}^{-1}\right)$ in $20 \mathrm{mM} \mathrm{KH}_{2} \mathrm{PO}_{4}$ buffer ( $\mathrm{pH} 7.8)$. For monitoring the molar ellipticity of $\mathrm{NaIC}$ bound to PCP, $8.7 \mu \mathrm{M}$ NalC was pre-incubated with $400 \mu \mathrm{M}$ $\mathrm{PCP}$ for $1 \mathrm{~h}$ at room temperature. This PCP concentration was sufficient to completely prevent NalC's binding to DNA. To assess reversibility of PCP binding, NalC-PCP was gel filtered through an Illustra NAP-5 column as described previously and retested. Far-UV CD scans (199-260 nm) for NalC, NalC-PCP and NalC after PCP removal were performed using a Jasco J-810 CD spectrophotometer (Jasco Analytical Instruments, Easton, MD, USA). Six scans were accumulated. The spectra of buffer alone or buffer with PCP were subtracted from the protein spectra. Thermal transitions of NalC, NalC-PCP and NalC after PCP removal were analysed between $30^{\circ} \mathrm{C}$ and $100^{\circ} \mathrm{C}$ (temperature was controlled by a Jasco PTC-423S) and readings were taken at $222 \mathrm{~nm}$. The rate of temperature increase was $1^{\circ} \mathrm{C} \mathrm{min}{ }^{-1}$. Thermal transition data were fitted with sigmoidal curves in SigmaPlot 10 to determine apparent median melting temperatures.

\section{Acknowledgements}

This work was supported by funding from the College of Engineering, University of Michigan for S.G. and supplies and, in part, by The National Institute of Health Grant GM065318 to U.J. We would like to thank H. Schweizer and K. Poole for $P$. aeruginosa strains.

\section{References}

Alekshun, M.N., and Levy, S.B. (2007) Molecular mechanisms of antibacterial multidrug resistance. Cell 128: 1037-1050.

Bengtson, P., Bastviken, D., de Boer, W., and Oberg, G. (2009) Possible role of reactive chlorine in microbial antagonism and organic matter chlorination in terrestrial environments. Environ Microbiol 11: 1330-1339.

Cann, J.R., and Hinman, N.D. (1976) Hummel-Dreyer gel chromatographic procedure as applied to ligand-mediated association. Biochemistry 15: 4614-4622.

Cao, L., Srikumar, R., and Poole, K. (2004) MexAB-OprM hyperexpression in NalC-type multidrug-resistant Pseudomonas aeruginosa: identification and characterization of the nalC gene encoding a repressor of PA3720-PA3719. Mol Microbiol 53: 1423-1436.

Cases, I., and de Lorenzo, V. (2005) Promoters in the environment: transcriptional regulation in its natural context. Nat Rev Microbiol 3: 105-118.

Chen, H., Hu, J., Chen, P.R., Lan, L.F., Li, Z.L., Hicks, L.M., et al. (2008) The Pseudomonas aeruginosa multidrug efflux regulator MexR uses an oxidation-sensing mechanism. Proc Natl Acad Sci USA 105: 13586-13591.

Daigle, D.M., Cao, L., Fraud, S., Wilke, M.S., Pacey, A., Klinoski, R., et al. (2007) Protein modulator of multidrug efflux gene expression in Pseudomonas aeruginosa. $J$ Bacteriol 189: 5441-5451.

Fiss, E.M., Rule, K.L., and Vikesland, P.J. (2007) Formation of chloroform and other chlorinated byproducts by chlorination of triclosan-containing antibacterial products. Environ Sci Technol 41: 2387-2394.

Ge, F., Zhu, L.Z., and Wang, J. (2008) Distribution of chlorination products of phenols under various $\mathrm{pHs}$ in water disinfection. Desalination 225: 156-166.

Graf, P.C.F., Martinez-Yamout, M., VanHaerents, S., Lilie, H., Dyson, H.J., and Jakob, U. (2004) Activation of the redoxregulated chaperone Hsp33 by domain unfolding. $J$ Biol Chem 279: 20529-20538.

Hellman, L.M., and Fried, M.G. (2007) Electrophoretic mobility shift assay (EMSA) for detecting protein-nucleic acid interactions. Nat Protoc 2: 1849-1861.

Hocquet, D., Berthelot, P., Roussel-Delvallez, M., Favre, R., Jeannot, K., Bajolet, O., et al. (2007) Pseudomonas aeruginosa may accumulate drug resistance mechanisms without losing its ability to cause bloodstream infections. Antimicrob Agents Chemother 51: 3531-3536.

Juhas, M., Eberl, L., and Tummler, B. (2005) Quorum sensing: the power of cooperation in the world of Pseudomonas. Environ Microbiol 7: 459-471.

Kerr, K.G., and Snelling, A.M. (2009) Pseudomonas aeruginosa: a formidable and ever-present adversary. J Hosp Infect 73: 338-344.

Li, X.Z., and Poole, K. (1999) Organic solvent-tolerant mutants of Pseudomonas aeruginosa display multiple antibiotic resistance. Can J Microbiol 45: 18-22.

Linares, J.F., Gustafsson, I., Baquero, F., and Martinez, J.L. (2006) Antibiotics as intermicrobial signaling agents instead of weapons. Proc Natl Acad Sci USA 103: 1948419489.

Lister, P.D., Wolter, D.J., and Hanson, N.D. (2009) Antibacterial-resistant Pseudomonas aeruginosa: clinical impact and complex regulation of chromosomally encoded resistance mechanisms. Clin Microbiol Rev 22: 582-610.

McLellan, L., Carvalho, M., Pereira, C.S., Hursthouse, A., Morrison, C., Tatner, P., et al. (2007) The environmental behaviour of polychlorinated phenols and its relevance to cork forest ecosystems: a review. J Environ Monit 9: 10551063.

Martinez, J.L. (2009) The role of natural environments in the evolution of resistance traits in pathogenic bacteria. Proc Biol Sci 276: 2521-2530.

Mena, K.D., and Gerba, C.P. (2009) Risk assessment of Pseudomonas aeruginosa in water. In Reviews of Environmental Contamination and Toxicology, Vol. 201. Whitcare, D.M. (ed.). New York: Springer, pp. 71-115.

Mesaros, N., Nordmann, P., Plesiat, P., Roussel-Delvallez, M., Van Eldere, J., Glupczynski, Y., et al. (2007) Pseudomonas aeruginosa: resistance and therapeutic options at the turn of the new millennium. Clin Microbiol Infect 13: 560-578.

Michalowicz, J., Bukowska, B., and Duda, W. (2008) The differences in phenolic content in rivers exposed and non- 
exposed to anthropogenic contamination. Chemosphere 71: 735-741.

Morita, Y., Cao, L., Gould, V.C., Avison, M.B., and Poole, K. (2006) nalD encodes a second repressor of the mexABoprM multidrug efflux operon of Pseudomonas aeruginosa. J Bacteriol 188: 8649-8654.

Muller, J.F., Stevens, A.M., Craig, J., and Love, N.G. (2007) Transcriptome analysis reveals that multidrug efflux genes are upregulated to protect Pseudomonas aeruginosa from pentachlorophenol stress. Appl Environ Microbiol 73: 4550-4558.

Myneni, S.C.B. (2002) Formation of stable chlorinated hydrocarbons in weathering plant material. Science 295: 10391041.

Neyfakh, A.A. (1997) Natural functions of bacterial multidrug transporters. Trends Microbiol 5: 309-313.

Orth, P., Schnappinger, D., Hillen, W., Saenger, W., and Hinrichs, W. (2000) Structural basis of gene regulation by the tetracycline inducible Tet repressor-operator system. Nat Struct Biol 7: 215-219.

Piddock, L.J.V. (2006) Multidrug-resistance efflux pumps not just for resistance. Nat Rev Microbiol 4: 629-636.

Ramos, J.L., Martinez-Bueno, M., Molina-Henares, A.J., Teran, W., Watanabe, K., Zhang, X.D., et al. (2005) The TetR family of transcriptional repressors. Microbiol Mol Biol Rev 69: 326-356.

Reichheld, S.E., Yu, Z., and Davidson, A.R. (2009) The induction of folding cooperativity by ligand binding drives the allosteric response of tetracycline repressor. Proc Natl Acad Sci USA 106: 22263-22268.

Rutala, W.A.R., Weber, D.J., and The Healthcare Infection Control Practices Advisory Committee (HICPAC) (2008) Guideline for Disinfection and Sterilization in Healthcare Facilities. Atlanta, GA: Center for Disease Control and Prevention.
Schobert, M., and Tielen, P. (2010) Contribution of oxygenlimiting conditions to persistent infection of Pseudomonas aeruginosa. Future Microbiol 5: 603-621.

Schweizer, H.P. (1998) Intrinsic resistance to inhibitors of fatty acid biosynthesis in Pseudomonas aeruginosa is due to efflux: application of a novel technique for generation of unmarked chromosomal mutations for the study of efflux systems. Antimicrob Agents Chemother 42: 394-398.

Song, J.K., Ahn, H.J., Kim, H.S., and Song, B.K. (2006) Molecular cloning and expression of perhydrolase genes from Pseudomonas aeruginosa and Burkholderia cepacia in Escherichia coli. Biotechnol Lett 28: 849-856.

Wagner, C., El Omari, M., and Konig, G.M. (2009) Biohalogenation: nature's way to synthesize halogenated metabolites. J Nat Prod 72: 540-553.

Weber, D.J., Rutala, W.A., and Sickbert-Bennett, E.E. (2007) Outbreaks associated with contaminated antiseptics and disinfectants. Antimicrob Agents Chemother 51: 42174224.

Wilke, M.S., Heller, M., Creagh, A.L., Haynes, C.A., Mclntosh, L.P., Poole, K., and Strynadka, N.C.J. (2008) The crystal structure of MexR from Pseudomonas aeruginosa in complex with its antirepressor ArmR. Proc Natl Acad Sci USA 105: 14832-14837.

\section{Supporting information}

Additional supporting information may be found in the online version of this article.

Please note: Wiley-Blackwell are not responsible for the content or functionality of any supporting materials supplied by the authors. Any queries (other than missing material) should be directed to the corresponding author for the article. 